\title{
Contact models verification by the finite element model updating method based on the calculation of the sensitivity coefficient
}

\author{
Vladimir Zhulev*, and Michail Kuts ${ }^{\dagger}$ \\ Bauman Moscow State Technical University, 105005 Moscow, Russia
}

\begin{abstract}
Vibrations, occurring in the cutting process, have significant effect on the accuracy of machined parts. Approaches, based on simplified simulation of parts and units, doesn't lead to reliable results in dynamic behavior simulation, because they suppose the ideal smoothness of contact surfaces. Detailed simulation of contact layer requires large computational costs and is difficult to perform for most of calculations in mechanical engineering. In this regard, an approach to model the contact layer as a third body with zero thickness, which parameters depends from many factors, particularly from contact pressure, is the most widespread. The experimental method based on the identification of the contact layer is considered in this paper, using the example of a free-fixed beam. The algorithm for determining the stiffness element in the contact layer is based on the sensitivity coefficients calculation. As verification of the method, a comparison was made with the theoretical stiffness model in the contact layer. It's investigated rigidity function in the region, depending on the natural frequency of the specify with different tightening forces. The obtained data can be used to correctly model the contact layer for dynamic problems.
\end{abstract}

\section{Introduction}

A several kinds of problems exist in mechanical engineering, in which necessary to simulate dynamical behaviour of constructions by finite elements methods, for example simulation of metal cutting process [1]. In this case errors of various kinds, which lead to the significant difference between model behavior and construction behavior, occur. One of the most important simplifications, that effects on model behavior, is contact between jointed surfaces of parts. Usually this area is ignored, by combining adjacent nodes of the model, or it's replaced by elements with unknown equivalent parameters. The model, thus obtained, needs correction and determination right contact parameters.

Theory of elasticity and modern computation methods allow to estimate stiffness of parts with any configuration precisely, under the assumption that the contact of the parts is topographically perfect. In fact, such contact can't be obtained even with ultraprecise

"Vladimir Zhulev: vlzh92@gmail.com

†Michail Kuts: kuts@,bmstu.ru 
methods of machining. Withal, several works [2 - 4], dedicated to the contact of rough surfaces, in which contact pressure and displacements relation approximate by power function, are exist. Limitation of this method are that it's needs a huge count of empirical data, for precise definition of contact layer pliability.

In works $[5,6]$ the method of calculation of contact stiffness, that takes into account materials of the contact parts and its surface roughness, was proposed. An advantage of this method is that it allows to define contact layer pliability on the design stage [7, 8]. A disadvantage of this method is scale effect, which is explaining by increasing influence of contact surface waviness.

However, we can use another approach to define contact stiffness - by using a method of finite elements model refinement. There are two methods of FE-model refinement: direct and iterative. Direct methods change matrix components, that used in finite element simulation $[9,10]$. A disadvantage of this methods is that parameters of the model can takes a value beyond physically reasonable range after the refinement.

Iterative methods haven't this disadvantage. Their main idea is that a target function is introduced, which is a measure of the discrepancy between the calculated and experimental values of natural frequencies, which can be minimized in several iterations by the methods of optimization theory. The method, based on coefficient of sensitivity, which calculation described in work [11], is a widespread. General equations are given in work [12].

In this article we consider two approaches for determining the equivalent rigidity of the contact layer of an assembled construction: by the method of determining based on a priory data about contacted surfaces $\left(1^{\text {st }}\right.$ method) and by the method of finite element model refinement based on calculation of the sensitivity coefficient using experimental modal analysis $\left(2^{\text {nd }}\right.$ method). The aim of this work is comparation of results of applying two approaches, that determine equivalent rigidity of elements in contact layer of assembled construction by example of cantilever beam.

\section{Description of the methods used}

\subsection{Determining of the contact stiffness using a priori data about contact surfaces (1st method)}

In the works $[5,6]$ the approach for determining contact layer rigidity. According to this approach normal and tangential rigidity $k_{a}$ and $k_{\tau}$ flat joint can be determined by equations:

$$
\begin{aligned}
k_{a} & =\frac{A}{\left(\lambda_{c} E+\lambda_{p} A\right)\left(1-\chi_{F a}\right)} \\
k_{a} & =\frac{A}{\left(\lambda_{c} E+\lambda_{p} A\right)\left(1-\chi_{F \tau}\right)}
\end{aligned}
$$

where $A$ and $I$ - a square and a moment of inertia of the joint, $\lambda_{c}-$ coefficient of the contact pliability of the joint, $\varepsilon$ - scale factor, depended from maximum dimension $l$ of contact surfaces and type of machining, $\lambda_{p}$ - pliability of assembled parts, $\chi_{F a}$ - load factor under the action of tensile force, $\chi_{F \tau}$ - load factor under the action of shearing force.

Coefficient of the contact pliability in equations (1) and (2) can be defined as follow:

$$
\lambda_{c}=\frac{c_{0} R a}{2 \sqrt{E^{*} p_{0}}}
$$


where $R a$ - arithmetical mean deviation of the assessed profile, $c_{0}$ - coefficient, which consider direction of contact surfaces roughness, $E^{*}=\left(\left(1-\mu_{1}\right)^{2} / E_{1}+\left(1-\mu_{2}\right)^{2} / E_{2}\right)^{-1}$ equivalent modulus of elasticity, in which $E$ - Yang modulus and $\mu$ - Poisson's ratio for each of parts, $p_{0}=z F_{\text {tight }} / A$ - initial contact pressure from tightening force $F_{\text {tight }}(z-$ number of bolts).

\subsection{The method of finite elements model refinement based on calculation of sensitivity coefficient using experimental modal analysis $\left(2^{\text {nd }}\right.$ method)}

\subsubsection{Calculation of sensitivity coefficient}

From the [13] it's known, that natural frequencies of model without damping can be calculated using Rayleigh relation:

$$
\omega_{i}^{2}=\frac{\mathbf{x}_{i}^{T} \mathbf{K} \mathbf{x}_{i}}{\mathbf{x}_{i}^{T} \mathbf{M} \mathbf{x}_{i}}
$$

where $\mathbf{K}$ - global stiffness matrix of system, $\mathbf{M}-$ global mass matrix of system, $\mathbf{x}_{i}-$ $i^{\text {th }}$ mode of the model vibration, $\omega_{i}-i^{\text {th }}$ natural frequency.

We can define the sensitivity coefficient natural frequency from changing stiffness of a selected finite element by differentiating of equation (4) by its stiffness and performing some transformations:

$$
\frac{\partial \omega_{i}}{\partial k_{j}}=\frac{\mathbf{x}_{i}^{T}\left(\mathbf{K}^{\prime k_{j}}-\omega_{i}^{2} \mathbf{M}^{\prime k_{j}}\right) \mathbf{x}_{i}}{2 \omega_{i} \mathbf{M}_{i}} \approx \frac{\mathbf{x}_{i}^{T} \mathbf{K}_{j}^{\prime} \mathbf{x}_{i}}{2 \omega_{i} \mathrm{M}_{i} k_{j}}=\frac{\Pi_{i j}}{\omega_{i} \mathbf{M}_{i} k_{j}}
$$

where $\mathbf{M}_{i}=\mathbf{x}_{i}^{T} \mathbf{M} \mathbf{x}_{i}-i^{\text {th }}$ modal mass, $k_{j}$ - stiffness of $j^{\text {th }}$ element, $\mathbf{K}^{k_{j}}, \mathbf{M}^{k_{j}}-$ local derivative of global stiffness and mass matrices by stiffness of $j^{\text {th }}$ element, $\mathbf{K}_{j}^{\prime}-$ the stiffness matrix of $j^{\text {th }}$ element, supplemented by zeros till dimension of the global stiffness matrix, $\Pi_{i j}$ - potential energy of deformation of $j^{\text {th }}$ element on the $i^{\text {th }}$ natural frequency.

In case when we need to tune the model on the $N$ lower natural frequencies and stiffness variation is allowed in $L$ finite elements, then it would be rational to combine sensitivity coefficients, determined by equation (5), in matrix with dimension $L \times N$, in which $j^{\text {th }}$ row present sensitivity coefficients, considered in eigen mode for

$$
\mathbf{T}=\left(\begin{array}{ccc}
\partial \omega_{1} / \partial k_{1} & \partial \omega_{2} / \partial k_{1} & \cdots \\
\partial \omega_{1} / \partial k_{2} & \partial \omega_{2} / \partial k_{2} & \cdots \\
\vdots & \vdots & \ddots
\end{array}\right) .
$$

\subsubsection{Algorithm of the stiffness refinement of the FE model}

Vector of natural frequencies can be represented as a function of stiffness parameters of some elements and we can perform the Taylor expansion to it: 


$$
\boldsymbol{\omega}(\mathbf{k})=\boldsymbol{\omega}\left(\mathbf{k}_{0}\right)+\frac{\boldsymbol{\omega}^{\prime}\left(\mathbf{k}_{0}\right)}{1 !}\left(\mathbf{k}-\mathbf{k}_{0}\right)+\frac{\boldsymbol{\omega}^{\prime \prime}\left(\mathbf{k}_{0}\right)}{2 !}\left(\mathbf{k}-\mathbf{k}_{0}\right)^{2}+\ldots \frac{\boldsymbol{\omega}^{(n)}\left(\mathbf{k}_{0}\right)}{n !}\left(\mathbf{k}-\mathbf{k}_{0}\right)^{n}
$$

Linearization of equation (7) by stiffness vector $\mathbf{k}_{0}$ leed to recurrsive expression for determining of unknown stiffness of the refinement model

$$
\mathbf{k}_{i+1}=\mathbf{k}_{i}+\left(\mathbf{T}_{i}\right)^{+} \cdot\left(\boldsymbol{\omega}(\mathbf{k})-\boldsymbol{\omega}\left(\mathbf{k}_{i}\right)\right),
$$

where $i$ - number of iteration, $\mathbf{k}_{i+1}$ - stiffness vector, which will be used on $(i+1)^{\text {th }}$ iteretion, $\mathbf{k}_{i}$ - stiffness vector, used on $i^{\text {th }}$ iteretion, $\left(\mathbf{T}_{i}\right)^{+}=\mathbf{T}_{i}^{T}\left(\mathbf{T}_{i}^{T} \mathbf{T}_{i}^{T}\right)^{-1}$ - pseudoinverse matrix of sensitivity coefficients, $\boldsymbol{\omega}(\mathbf{k})$ - vector of experimental values of the frequencies, $\boldsymbol{\omega}\left(\mathbf{k}_{i}\right)$ - vector of the frequencies, received on $i^{\text {th }}$ iteretion of calculation.

\subsubsection{Algorithm of the stiffness refinement}

For minimization disparity between natural frequency, obtained in simulation, and natural frequency, obtained in modal testing, it needs to define equivalent stiffnesses of the model. For correct performing of this determining the iterative algorithm of refinement is used.

If the disparity natural frequencies of the model and real object is unacceptable, the sensitivity coefficients (5) are calculated, then computation of stiffness of refinement elements are performed using equation (8). Then values of natural frequencies are calculated for model with changed properties. The process is repeated till disparity reach an acceptable value.

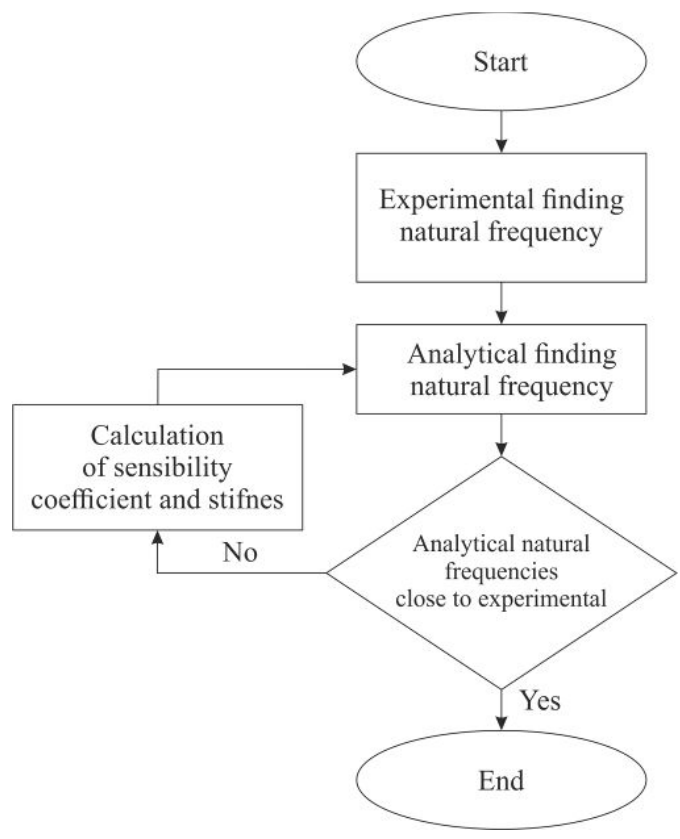

Fig. 1. Block-scheme of the finite element model updating algorithm based on calculating of the sensitivity coefficient. 


\section{Description of investigated object and research method}

\subsection{Identification of modal parameters by vibroimpulsive test results}

Identification of dynamic parameters was conducted on test sample, shown on fig. 2-a. Investigated sample represents an aluminum beam (pos. 1), which $L \times W \times H$ dimensions are $200 \times 45 \times 34 \mathrm{~mm}$, attached to the foundation by four M8x60 bolts (pos. 4). Dimensions of the foundation are $L \times W \times H=124 \times 80 \times 34 \mathrm{~mm}$. Dimensions of the contact surface between the foundation and the beam are $80 \times 45 \mathrm{~mm}$. The testing sample fixed on the steel support plate (pos. 3). The tightening torque of bolts pos.4 was varied due to experiment (see table 1). For experimental identification of dynamical properties of the test sample the vibroimpulsive test was carried out. The testing sample picture shown on the fig. $2-b$. The vibroimpulsive impact was performed with KISTLER dynamometric hummer, and data was measured by Brüel \& Kjær with farther acquiring by ADC Quattro made by Data Physics.

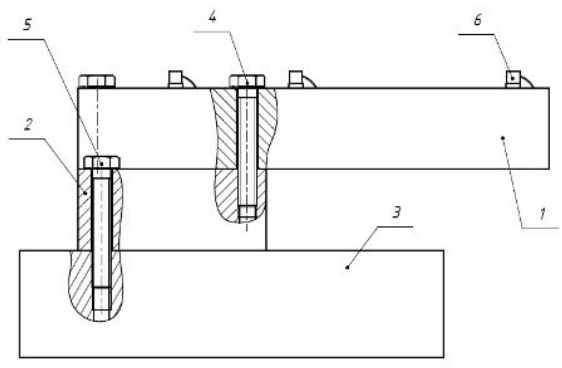

a)

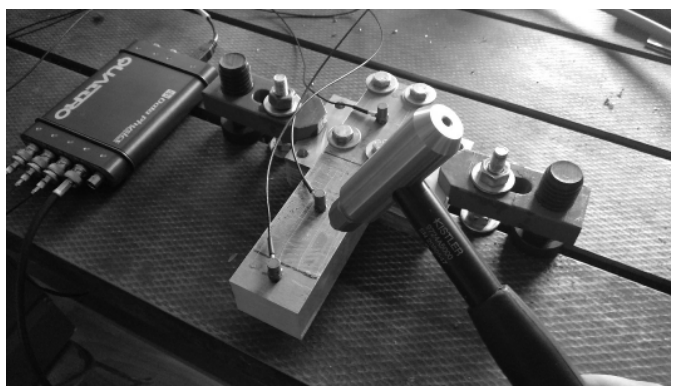

b)

Fig. 2. Scheme $(a)$ and picture $(b)$ of testing sample: 1 - beam; 2 - foundation; 3 - support plate; 4 bolts for beam fixing to the foundation; 5 - bolts for foundation fixing; 6 -accelerometers

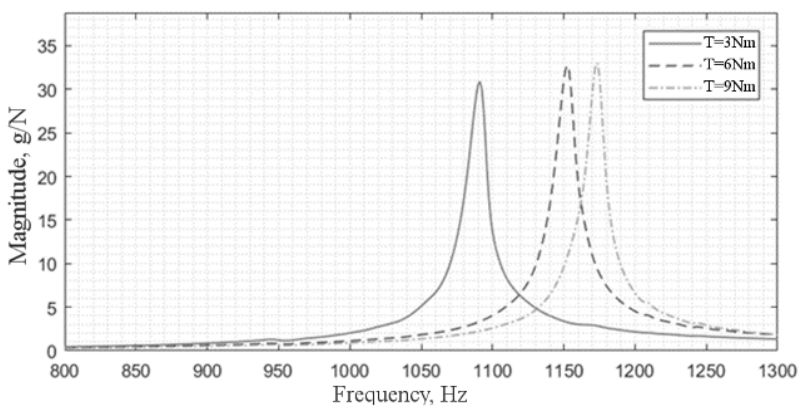

Fig. 3. Magnitude spectrum of free vibration of cantilever beam with various tightening forces and roughness $\mathrm{Ra}=10.2 \mathrm{mkm}$

\subsection{Finite element model description}

The model was made in CAE system NX Nastran and represents two parallelepipeds, which are located at a distance $\sim 0.1 \mathrm{~mm}$ from each other. These parallelepipeds covered by regular mesh of tetrahedral elements of the second order. Corresponding nods of parallelepipeds in the contact area connected with 54 springs (see fig. 4). Each of springs in the contact area has a unique rigidity. Bottom bound of the lower parallelepiped is fixed. 


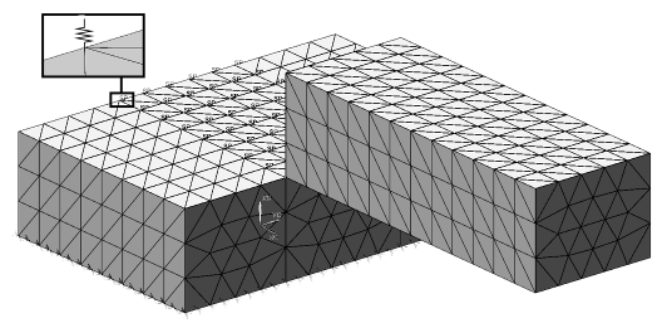

Fig. 4. Representation of finite element model (part of the beam under the contact was hided)

\section{Results of algorithms applying}

\subsection{Overall result}

Results of applying the approaches for stiffness determining, described above, shown in table 1 .

Table 1. Results of applying the approaches for stiffness determining.

\begin{tabular}{|c|c|c|c|c|c|}
\hline $\begin{array}{l}\text { \# of } \\
\text { measu- } \\
\text { rement }\end{array}$ & $\begin{array}{l}\text { Tightening } \\
\text { torque, } \mathrm{Nm}\end{array}$ & $\begin{array}{l}\text { Experimental } \\
1^{\text {st }} \text { natural } \\
\text { frequency, } \mathrm{Hz}\end{array}$ & $\begin{array}{c}\text { Contact } \\
\text { stiffness, } \\
\text { obtained by } \\
(1), \mathrm{N} / \mathrm{mm}\end{array}$ & $\begin{array}{l}\text { Overall contact } \\
\text { stiffness, obtained in } \\
\text { result of } \mathrm{FE} \mathrm{model} \\
\text { refinement, } \mathrm{N} / \mathrm{mm}\end{array}$ & Stiffness error \\
\hline 1 & 3 & 1091 & $2.36 \times 10^{6}$ & $4.01 \cdot 10^{6}$ & 41,2 \\
\hline 2 & 6 & 1153 & $2.95 \times 10^{6}$ & $4.89 \cdot 10^{6}$ & 39.7 \\
\hline 3 & 9 & 1175 & $3.19 \times 10^{6}$ & $5.32 \cdot 10^{6}$ & 40.0 \\
\hline
\end{tabular}

\subsection{Results of applying the algorithm Captions/numbering}

The algorithm of FE model stiffness, based on sensitivity coefficient calculation, was implemented using programming language MATLAB. CAE NX Nastran was used as FEM solver. Interpretation of obtained results was implemented in system WolframAlpha 9.0.

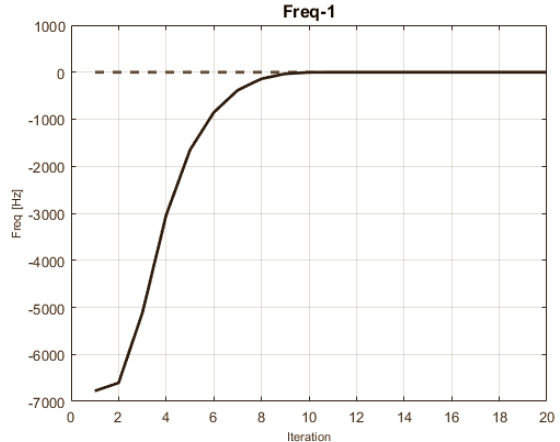

a)

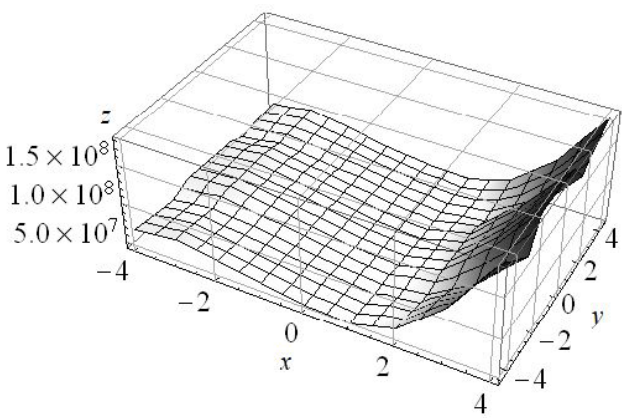

b)

Fig. 5. Illustration of the $2^{\text {nd }}$ algorithm work in case tightening torque Ttigh $=3 \mathrm{Nm}$, roughness parameter $\mathrm{Ra}=10.2 \mathrm{mkm}$ and initial stiffness value $\mathrm{k} 0=2,36 \times 10^{6} \mathrm{~N} / \mathrm{mm}$ : plot $(a)$ of disparity between 1 st natural frequencies, obtained by testing and by calculation, and number of iteration relation; plot of example stiffness distribution on the contact area $(b)$. 
During use of the refinement algorithm the series of similar results was obtained. The result of stiffness identification obtained with tightening torque $T_{\text {tigh }}=3 \mathrm{Nm}$ and roughness parameter $R a=10.2 \mathrm{mkm}$ are considered below more in detail.

The graph of the convergence of the $1^{\text {st }}$ natural frequency depending on iteration number shown on the fig. 5 - $a$.

The refinement algorithm application allows to determine the stiffness distribution in the contact area (see fig. $5-b$ )

\subsection{Investigation of correlation between result and initial stiffness}

Investigation of result stiffness, obtained by method of FE model refinement base on calculation of sensitivity coefficient, and initial stiffness value correlation was made. On the fig. 7 plot of result stiffness and initial contact stiffness relation and disparity of natural frequency value and initial stiffness relation are shown.

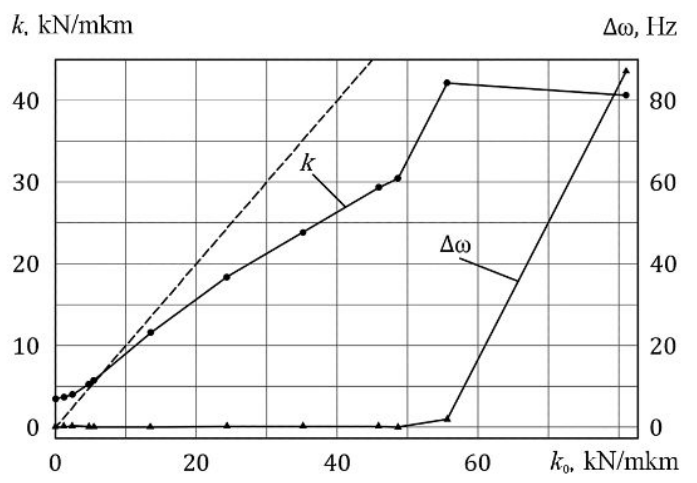

Fig. 7. Plot of result stiffness(k) and disparity between natural frequencies, obtained by the tests and by the calculations, obtained at various values of initial stiffness

\section{Analysis of the results}

Relation between result stiffness, showed on fig. 7, shows a value of the initial stiffness, with which method 2 don't change resulting contact stiffness (intersection point with dashed line). In cases if initial stiffness is different from it value, model updating algorithm will vary elements stiffness and at the first time those, which have higher deformation energy (see fig. 8). Although, the plots on the fig. 7 shows, that in case if initial stiffness differ by order (value higher than $50 \mathrm{kN} / \mathrm{mkm}$ ), then the $2^{\text {nd }}$ method doesn't allow to determine the contact stiffness. For fast convergence of the $2^{\text {nd }}$ method the initial stiffness needs to be close to the actual stiffness. This evaluation can be obtained by the $1^{\text {st }}$ method.

The natural frequency convergences with a preset accuracy in the $2^{\text {nd }}$ method, but as the result the obtained stiffness distribution has local peaks. These peaks can be divided on two typical types:

1. The peak on the edge of contact plate (domain $2 \leq x \leq 4,5$ and $-4 \leq y \leq 4$ ) can be explained by algorithm feature, which change the domains with the high deformation energy (see fig. 8) at first.

2. The peaks local to the bolts can be explained by phenomenon of stiffness distribution inside the pressure cone $[14,15,16]$. Wherein, in the hole places there are the sinks (domain $-4,5 \leq x \leq-2$ and $-4 \leq y \leq 4)$. 


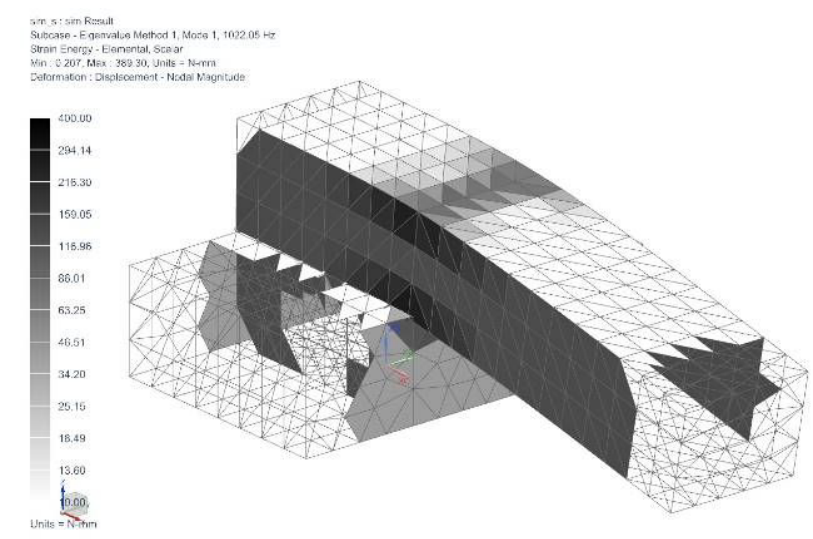

Fig. 8. Illustration of deformed model on the 1 st iteration (darker region corresponds to the region with higher deformation energy)

The result, presented in the table 1, shows that maximum error between average stiffness values, obtained by the two methods, is $40 \%$. But the natural frequency error, calculated with stiffness value obtained by the $1^{\text {st }}$ method, is not higher than $7 \%$. This can be explained by fact, that $2^{\text {nd }}$ method changes stiffness of elements with high deformation energy, which effects on the average stiffness value.

\section{Conclusion}

1. Maximum error of natural frequency calculation by method of stiffness determining based on a priory data is $7 \%$. This shows, that this method can be used for preliminary estimation of the natural frequencies, but it's not acceptable for accurate simulation of the system dynamic behavior;

2. Accurate simulation of dynamic behavior of the construction needs to conduct experimental refinement of stiffness parameters by finite element model updating, based on the sensitivity coefficient calculation. For fast convergence we can use the initial stiffness obtained by the $1^{\text {st }}$ method;

3. Accuracy of the method of stiffness determining based on a priory data can be increased by considering the stiffness distribution in the local of the bolts.

4. For more receiving more accurate distribution more parameters, as higher values of the natural frequencies, need to be considered in the model updating method, based on the sensitivity coefficient calculation.

\section{References}

1. S.A. Voronov, I.A. Kiselev, Mechanical engineering and engineer education, 2 (51), 9 (2017)

2. Z. M. Levina, D. N. Reshetov, Kontaktnaya dzheskost' mashin [Contact stiffness of machines] (Mashinostroenie, Moskow, 1971)

3. N. B. Demkin, E.V. Rizhov, Kachestvo poverhnosti I kontakt detaley mashin [Surface quality and contact of machine parts] (Mashinostoenie, Moscow 1981)

4. E.V. Rizhov, Kontaktnaya dzhestkost' detaley machin [Contact stiffness of the machine parts] (Mashinostroenie, Moscow, 1966)

5. A. S. Ivanov, Vestnik Mashinostroeniya, 7, 34 (2007) 
6. A.S. Ivanov, V.V. Izmailov, Friction \& Lubrication in Machines and Mechanisms, 7, 34 (2007)

7. M. M. Ermolaev, Russian Engineering Research, 36(5), 355 (2016)

8. A. S. Ivanov, M.M. Ermolaev, Vestnik Mashinostroeniaya, 10, 54 (2010)

9. M. Baruch, I.Y. Bar Itzhack, AIAA Journal, 17, 927 (1979)

10. A. Berman, E.J. Nagy, AIAA journal, 21, 1168 (1983)

11. Géradin M., Rixen D.J. Mechanical vibrations: theory and application to structural dynamics (John Wiley \& Sons, UK, 2014)

12. M. Friswell, J.E. Mottershead, Finite element model updating in structural dynamics, 38 (Springer Science \& Business Media, 2013)

13. V. L. Biderman, Teoriya mehanicheskih kolebaniy [Theory of mechanical vibrations]. (Lenand, Moscow, 2017)

14. I.A. Birger, G.B. Iosilevich, Rezbovie I flancevie soedineniya [Bolted and flanged joints] (Mashinostroeniye, Moscow, 1990)

15. Y. Ito, Modular design for machine tools (McGraw Hill Professional, 2008)

16. Plock R. Die, Ind. Anz., 93 (27), 571 (1971) 